\title{
Velocity autocorrelation function in supercooled liquids: Long-time tails and anomalous shear-wave propagation
}

\author{
H. L. Peng,,${ }^{1,2, *}$ H. R. Schober, ${ }^{3}$ and Th. Voigtmann ${ }^{1,4}$ \\ ${ }^{1}$ Institut für Materialphysik im Weltraum, Deutsches Zentrum für Luft- und Raumfahrt (DLR), 51170 Köln, Germany \\ ${ }^{2}$ Institute for Materials Research, Tohoku University, Sendai 980-8577, Japan \\ ${ }^{3}$ Peter Grünberg Institut, Forschungszentrum Jülich, D-52425 Jülich, Germany \\ ${ }^{4}$ Department of Physics, Heinrich-Heine-Universität Düsseldorf, Universitätsstraße 1, 40225 Düsseldorf, Germany
}

(Received 1 September 2016; published 15 December 2016)

\begin{abstract}
Molecular dynamic simulations are performed to reveal the long-time behavior of the velocity autocorrelation function (VAF) by utilizing the finite-size effect in a Lennard-Jones binary mixture. Whereas in normal liquids the classical positive $t^{-3 / 2}$ long-time tail is observed, we find in supercooled liquids a negative tail. It is strongly influenced by the transfer of the transverse current wave across the period boundary. The $t^{-5 / 2}$ decay of the negative long-time tail is confirmed in the spectrum of VAF. Modeling the long-time transverse current within a generalized Maxwell model, we reproduce the negative long-time tail of the VAF, but with a slower algebraic $t^{-2}$ decay.
\end{abstract}

DOI: 10.1103/PhysRevE.94.060601

One of the simplest parameters for measuring liquid dynamics is the diffusion coefficient, which can be calculated from the mean-square displacement or from the single-particle velocity autocorrelation function (VAF) through the Green-Kubo relation $[1,2]$. In dilute fluids, where the correlation between binary collisions can be neglected, the time-dependent VAF decays exponentially. This has been validated by the kinetic theory for gases or granular media and Brownian dynamics $[1,3]$. It came as a surprise when Alder and Wainwright for the first time reported that the long-time tail of the VAF decays algebraically as $t^{-d / 2}$, where $d$ is the dimension of the system [4-6]. This long-lasting correlation is attributed to the well-known hydrodynamic vortex, or backflow, that supports the initial motion and develops a persistent long time tail $[7,8]$.

At high densities the initial direction of motion of an atom is, on average, soon reversed as the atom feels the surrounding neighbors, as a negative region. For the reversed velocity a $t^{-5 / 2}$ decay has been reported for a hard-sphere fluid [9]. Interestingly, the same algebraic decay is observed in a Lorentz gas, where the long-time tail has been shown both theoretically [10] and by computer simulation (at low obstacle density) to decay as $-t^{-d / 2-1}$ [11]. A plausible conjecture is that they would have the same physical mechanism. Since the dynamic heterogeneity (both in space and time) manifests itself in the dynamics of high volume fraction of hard-sphere and of supercooled liquids [12-14], groups of immobile atoms might play the role of the fixed scatterers for mobile atoms as in the Lorentz gas. They then account for the negative long-time tail [9]. Actually frozen scatterers are not essential for the emergence of negative long-time tails. A more general approach using coarse graining has theoretically predicted a negative $t^{-d / 2-1}$ decay [15-17].

There are two mechanisms for the decay of the singleparticle momentum in atomic or molecular liquids: diffusion and sound propagation. By diffusion the momentum of a tagged atom is transferred into a region of typical length $l=$ $\sqrt{D t}$ ( $D$ is the diffusion coefficient) and the velocity decays

${ }^{*}$ Corresponding author: hailong.peng@imr.tohoku.ac.jp as $v_{i}(t) \sim v_{i}(0) /\left(\rho l^{3}\right) \propto t^{-3 / 2}$, which is the classical long-time tail. In accordance with this mechanism, the long-time decay of the VAF has been reported as $\propto t^{-3 / 2}$ in momentumconserving and as $\propto t^{-1}$ in momentum-nonconserving granular fluids [18]. The initial momentum of a tagged atom can also be rapidly carried away by propagating sound waves. Usually, this contributes only to the short time decay of the VAF [19]. As the system is confined, the long-time hydrodynamic tail is expected to be lost and this mechanism becomes important. A transition from a positive to a negative $t^{-\mu}$ decay has been reported in the confined systems, with the exponent $\mu$ depending on the confining geometry [20]. Diffusive sound-wave modes have been found to account for the negative long-time tail in this situation [20].

In viscous liquids, the competition between these two mechanisms is responsible for the sign change of the long-time tails. One reason for this is that transverse sound waves are overdamped at high temperatures, but underdamped in supercooling. The ability of supporting long-distance shearwave propagation in supercooled liquids [21,22] enhances the momentum transfer capability of the sound wave for long times. Recently, a negative $t^{-3 / 2}$ long-time tail was observed in polymers at the zero friction limit, as predicted by the viscoelastic hydrodynamic interaction model [23]. In this scenario, viscoelasticity is important for the velocity fluctuations in supercooled liquids.

To address this question in atomic liquids, we performed molecular dynamics (MD) computer simulations for a Lennard-Jones potential. The physics exposed by this work includes the following: (1) A strong system size dependence of the long-time tail of the VAF is observed and found to be caused by long-lived transverse waves in supercooled liquids. (2) In supercooled liquids a negative long-time tail of the VAF is found, decaying as $t^{-5 / 2}$. (3) By a generalized Maxwell model for shear-wave propagation, the negative long-time tail is reproduced, but with a slower algebraic decay: $t^{-2}$.

The MD simulation is done for a Lennard-Jones system with Kob-Andersen parameters and composition $A_{80} B_{20}$ [24] using the open software LAMMPS [25]. To frustrate vacancy nucleation, a fixed pressure $(=5.0)$ is exerted on the system 


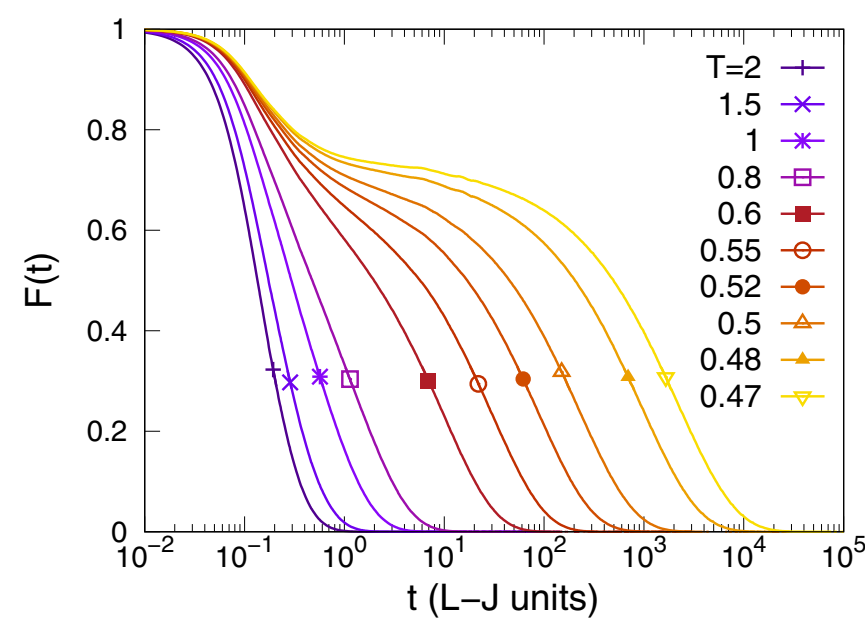

FIG. 1. Temperature dependence of self-intermediate scattering function $F(t)$, which is defined as $F(t)=\left\langle\exp \left[i\left(\mathbf{r}_{i}(t)-\mathbf{r}_{i}(0)\right) \cdot \mathbf{q}_{0}\right]\right\rangle$, where $q_{0}$ is the first peak position in the static structure factor.

at all temperatures. The self-intermediate scattering function for this system is shown in Fig. 1. A clear second step relaxation is observable at $T<0.8$, indicating the slow structural relaxation in the supercooled liquid state. Fitting the temperature-dependent tracer diffusivity, we find the mode-coupling theory crossover temperature at $T \approx 0.46$. Microcanonical ensembles are used to equilibrate the system and to collect data. No temperature or pressure drift is observed during this process. Three different systems sizes are used for finite-size effect investigations. The total number of atoms are $N=3000,24000,100000$. If not specified otherwise, the data given below are for the largest system, i.e., $N=100000$.

Figure 2 shows the calculated VAF, $Z(t)=\frac{1}{3}\left\langle\mathbf{v}_{i}(t) \mathbf{v}_{i}(0)\right\rangle$. At high temperature, e.g., at $T=2$, the tail of $Z(t)$ stays positive, while for $T=1.5, Z(t)$ crosses the abscissa four times already for short times up to $t=1$ and eventually approaches zero from above. This positive decay is well described as $t^{-3 / 2}$ [indicated by the dashed line in Fig. 2(a)],

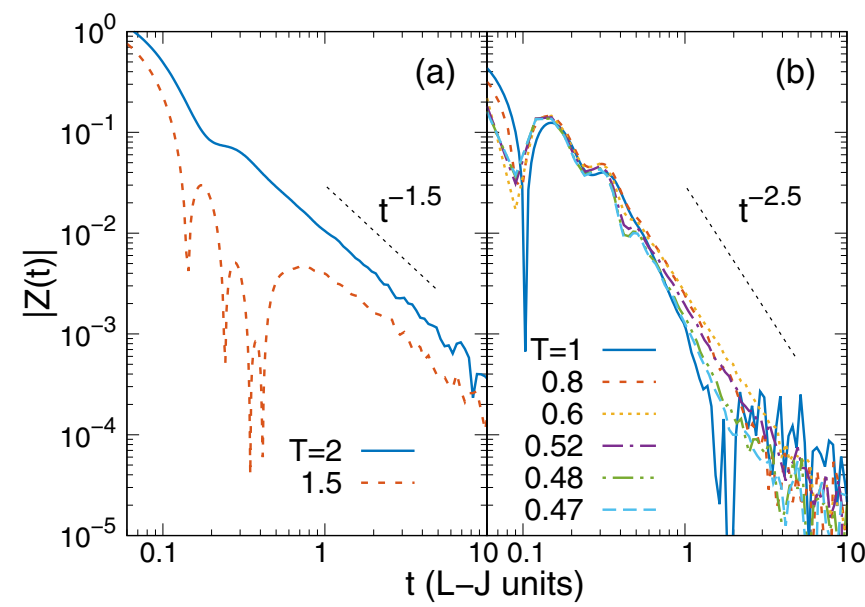

FIG. 2. Absolute value of the velocity autocorrelation function $Z(t)$ as function of time, high temperatures (a) and lower temperatures (b). Dips in the curves indicate sign changes of $Z(t)$.

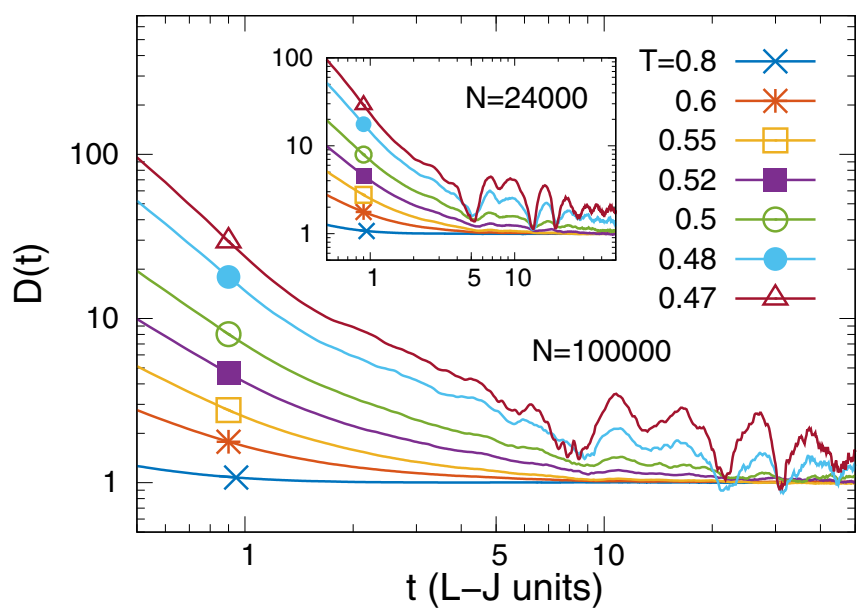

FIG. 3. Normalized time-dependent diffusivity, $D(t)=$ $\int_{0}^{t} d t Z(t) / D_{\mathrm{msd}}$, in supercooled liquids. Two different system sizes are shown, i.e., $N=100000$ and $N=24000$ (inset). Regions where $D(t)$ increases indicate positive values of the VAF, decreasing ones indicate negative values of the VAF. The oscillations of $D(t)$ show a clear system-size dependence.

indicating that the motion of atoms is collective as induced by the hydrodynamic vortex [7]. At relative low temperatures, i.e., $T<1$ [see Fig. 2(b)], the negative tails do not show a consistent $t^{-5 / 2}$ decay as predicted by the theory [15-17]. Instead these long-time tails show a temperature-dependent behavior. Similar behavior can also be seen at high packing fractions of hard-sphere liquids [9]. This indicates an unstable long-time tail of $Z(t)$, or the destroyed tail in high-density liquids, in contrast to the hydrodynamic situation and also the results reported in other systems [9-11].

To clarify this problem, we investigate the normalized time-dependent diffusivity, $D(t)=\int_{0}^{t} d t Z(t) / D_{\text {msd }}$, shown in Fig. 3. $D_{\text {msd }}$ is the diffusion coefficient calculated from the mean-square displacement: $D_{\mathrm{msd}}=\lim _{t \rightarrow \infty}\langle| \mathbf{r}_{i}(t)-$ $\left.\left.\mathbf{r}_{i}(0)\right|^{2}\right\rangle / 6 t$. The time evolution of $D(t)$ shows a surprising oscillatory behavior at low temperatures. Since an increase of $D(t)$ corresponds to a positive value of $Z(t)$ and vice versa, the oscillatory behavior of $D(t)$ indicates that the sign of $Z(t)$ changes many times. For the $N=100000$ system, we find the first minimum position at $t_{m} \approx 9$. If we reduce the box size, i.e., for the $N=24000$ system, the first minimum position shifts to $t_{m}=5.3$ (see inset of Fig. 3). A constant ratio $L / t_{m}$ ( $L$ is the box size) is found for all system sizes investigated. This indicates a wave is crossing the period boundary with a propagation velocity $L / t_{m} \approx 5.0$, close to the velocity of the transverse current $(\approx 4.4$, calculated from its dispersion relation). We notice that the valleys of oscillation are almost temperature independent, which is due to the weak temperature dependence of the acoustic velocity.

This result shows that the long-time tail of $Z(t)$ is seriously influenced by shear-wave propagation. When a shear wave propagates from a tagged atom to its periodic image, the velocity of the atom is reversed. As the shear wave crosses the boundary of the simulation box many times, the atom will move backward and forward repeatedly, i.e., the atoms rattle inside their cage. At low temperatures, the effect of 

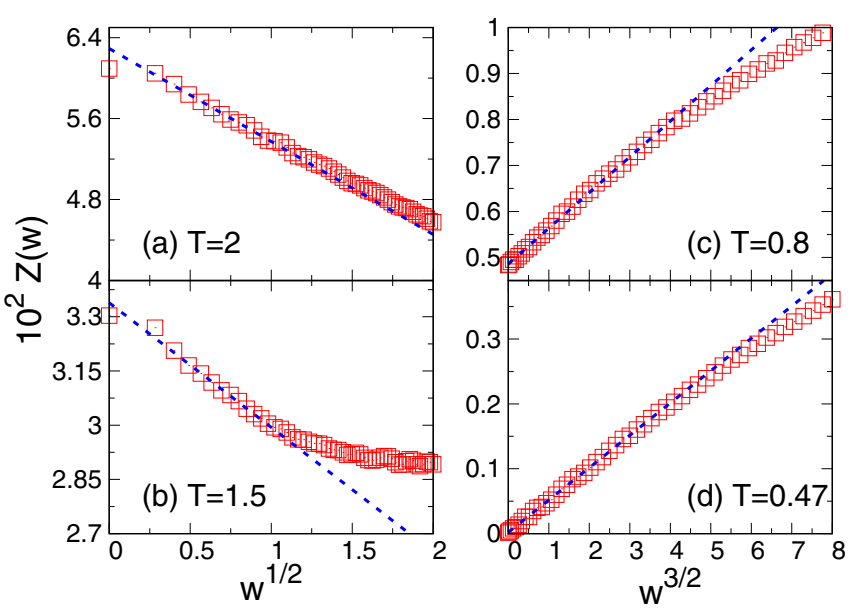

FIG. 4. Spectrum of the velocity autocorrelation function $Z(w)$. Dashed straight lines show linear fits of the low-frequency data. Left: normal liquids, where $Z(w) \propto w^{1 / 2}$ at low frequencies. Right: supercooled liquids, where $Z(w) \propto w^{3 / 2}$ in the low-frequency limit.

shear-wave propagation becomes so strong that the long-time tail of the VAF is eventually destroyed, as shown in Fig. 2(b). This makes it difficult to determine the long-time behavior of the VAF by computer simulation. In an infinite system or in the usual experiments, the effect of shear-wave propagation across period boundaries will disappear.

As an alternative, we investigate the Fourier transform of the VAF: $Z(w)=\frac{2}{\pi} \int_{0}^{\infty} d t Z(t) \cos (w t)$. Since here we are interested in the long-time behavior of $Z(t)$, we only show the low-frequency part of $Z(w)$ in Fig. 4. In normal liquids $(T \geqslant 1.5), Z(w) \propto w^{1 / 2}$ is found at low frequencies, which corresponds to $Z(t) \propto t^{-3 / 2}$ in time-space. The $w^{1 / 2}$ region shrinks at $T=1.5$ compared with $T=2$, which indicates that the hydrodynamic tail weakens with decreasing temperature. In low-temperature liquids $(T \leqslant 0.8), Z(w) \propto w^{3 / 2}$ at small $w$ is observed, which corresponds to $Z(t) \propto t^{-5 / 2}$ in the long time limit. We checked that a variation of the exponent for $3 / 2$ does not give a better linear fit in the low-frequency region. Compared to $T=0.8$, the region $Z(w) \propto w^{3 / 2}$ at $T=0.46$ has slightly grown, which would be a hint that the $t^{-5 / 2}$ longtime tail strengthens upon undercooling.

We have noticed that the transverse acoustic wave is important for the long-time behavior of the VAF. Actually, one of the hallmarks of supercooled liquids is the anomalous transverse-wave propagation: shear waves can propagate over long distances for macroscopic wave vectors, as in elastic media, but attenuate quickly for microscopic wave vectors, as in viscous liquids, [22]. Possibly there is a connection between this anomalous shear-wave propagation and the long-time decay behavior of the VAF.

Figure 5 shows the spectrum of the transverse current calculated as $C_{T}(w)=\frac{2}{\pi} \int_{0}^{\infty} d t C_{T}(t) \cos (w t)$, where $C_{T}(q, t)=$ $q^{2}\left\langle\mathbf{j}_{\mathbf{q}}^{\perp}(t) \mathbf{j}_{\mathbf{q}}^{\perp}(0)\right\rangle / N$ is the transverse current [1]. Here, $\mathbf{j}_{\mathbf{q}}=$ $\sum_{i}^{N} \mathbf{u}_{i}(t) \exp [-i \mathbf{q} \cdot \mathbf{r}(t)]$ and $\perp$ indicates that the wave vector $\mathbf{q}$ is perpendicular to the velocity $\mathbf{u}$. Clear sound peaks are observed at all shown temperatures. These peaks are characterized by position (corresponding to the propagation frequency), and half width of the peak (its inverse gives the
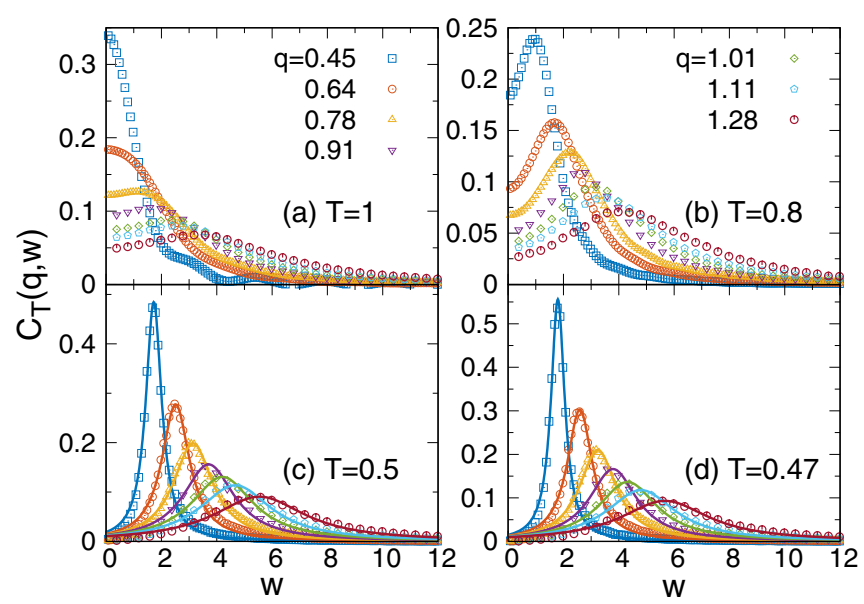

FIG. 5. Transverse current spectrum $C_{T}(q, w)$ in slightly supercooled liquids, (a) $T=1$ and (b) $T=0.8$, and highly supercooled liquids, (c) $T=0.5$ and (d) $T=0.46$. The data are for the $N=3000$ system. We do not find any visible finite-size effect for the transverse current at the same $q$ values. In (c) and (d), solid lines are the fits by Eq. (1).

attenuation rate of the mode). From temperature $T=1$ to $T=0.8$ [Figs. 5(a) and 5(b)], we see a gradual emergence of the peaks at finite frequency. At lower temperatures [Figs. 5(c) and 5(d)], these peaks become much sharper, indicating the propagation behavior of these small $\mathbf{q}$-vector modes.

The viscoelastic behavior of the transverse current can be characterized by the generalized Maxwell model with the Lorentzian spectrum [22,26-28]:

$$
C_{T}(q, w) \propto \frac{\Gamma(q)}{[w-\Omega(q)]^{2}+\Gamma(q)^{2}},
$$

where $\Omega(q)$ is the peak position and $\Gamma(q)$ the half width of the broadening. The fits are shown in Figs. 5(c) and 5(d) by solid lines.

To quantitatively investigate the influence of the viscoelastic modes on the long-time behavior of the VAF, we employ the expression of the velocity autocorrelation function derived by dynamic theory or the velocity field approach method $[1,26,29]$ :

$$
Z(t)=\frac{1}{3 k_{B} T m} \frac{1}{(2 \pi)^{3}} \int d \mathbf{q}\left[C_{L}(q, t)+2 C_{T}(q, t)\right] F(q, t),
$$

where $C_{L}(q, t)$ is the longitudinal current, which decays exponentially [1] with time, mainly contributes to the highfrequency part of $Z(w)$ [29], and can be neglected in the long time limit. $F(q, t)$ is the self-intermediate scattering function. Compared to the velocity field, the density fluctuation decays much slower, therefore $F(q, t)$ can be approximated as unity. With these approximations, at long times the VAF can be rewritten as

$$
Z(t) \propto \int d \mathbf{q} C_{T}(q, t) .
$$

To verify the role of the viscoelasticity, we calculate $C_{T}(q, t)$ from the fits of $C_{T}(q, w)$ via Eq. (1). In this calculation, the major contribution sustained in $Z(t)$ is the viscoelasticity. 


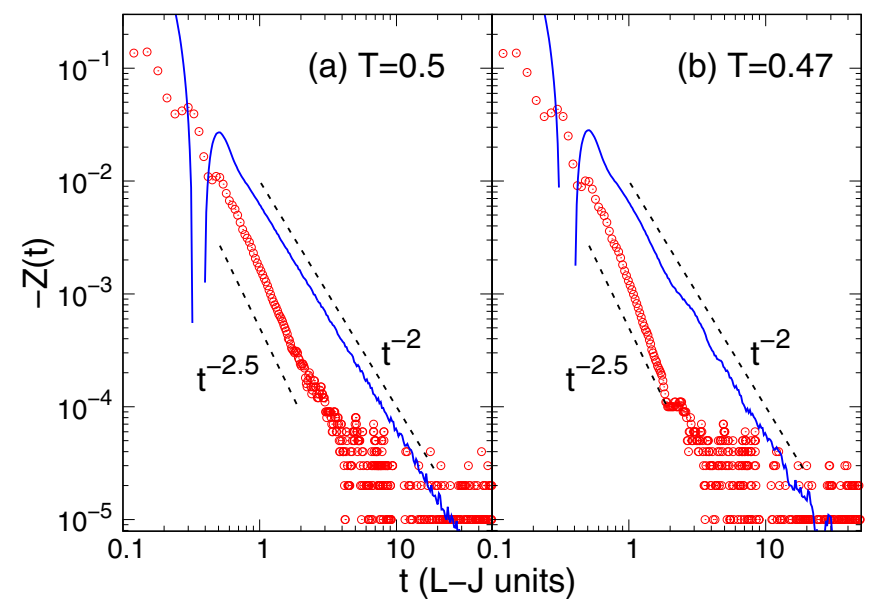

FIG. 6. Log-log plot of the negative value of the velocity autocorrelation function. Red circles: $Z(t)$ calculated from the definition, the same as in Fig. 2. Solid blue lines: $Z(t)$ calculated from Eq. (2), with the transverse current taken from the fit by the viscoelastic model, i.e., Eq. (1).

Figure 6 shows $Z(t)$ calculated from the viscoelastic model, together with the data calculated from its definition (the same data as in Fig. 2). Clearly, the negative long-time tails are reproduced by the viscoelastic model. Compared with the data $Z(t)$ directly calculated from its definition, the viscoelastic model gives some differing results. First, the oscillations which destroy the long-time tails disappear. As shown in Fig. 3, the first dip point should be around several time units. It is missing in the viscoelastic model. This gives rise to a longer time range of algebraic decay. Second, the viscoelastic model predicts a negative long-time decay as $t^{-2}$, which is slightly slower than the $t^{-5 / 2}$ decay when all contributions are included. This slower decay indicates that the viscoelastic fitting underestimates some fast decay channels, e.g., hydrodynamic modes and/or the interaction between hydrodynamics and viscoelastics. Third, the algebraic decay $t^{-2}$ is independent of temperature, which agrees with the independence of the $t^{-5 / 2}$ decay found in Fig. 4 for the supercooled liquids.

In undercooling, dynamic heterogeneity manifests itself in temporal and spatial fluctuations of the atomic displacements [12-14]. Groups of immobile atoms could form relative compact clusters at appropriate time scales [30]. Connection of these compact clusters enables the liquid to support the propagation of acoustic shear waves, and at the same time frustrates the diffusion transport like in the confining geometry [20]. As discussed earlier, the diffusion process accounts for the classic positive long-time tail, while acoustic propagation for the negative tail. Dynamic heterogeneity could promote a transition from the positive to the negative tail.

In normal liquids, it is known that the $t^{-3 / 2}$ law is the leading term of the asymptotic expansion of VAF [31,32], and the subleading term is essential for the intermediate-time behavior of the VAF [33]. The $t^{-5 / 2}$ decay would also be one of the infinite terms in the asymptotic expansion in supercooled liquids. Unfortunately, theory for this issue in supercooled liquids is still lacking.

The system investigated here is a binary mixture. But we do not find any significant difference of the long-time tail behavior (e.g., algebraic decay in Fig. 2) between particles $\mathrm{A}$ and $\mathrm{B}$, as well as of the sound-wave propagation behavior (e.g., dip positions in Fig. 3). Because the relevant vibrations here are acoustic modes (rather than optic ones), an atomicspecies-dependent behavior is not expected.

In conclusion, we observed the classical $-3 / 2$ power-law decay of the VAF at high temperatures. Upon supercooling, a transition of this positive tail to a negative $-5 / 2$ power-law decay is found, which agrees with findings in hard-sphere fluids [9]. In highly supercooled liquids, elasticity becomes so strong that the long-time tail of VAF is nearly destroyed by the transfer of shear waves across the period boundary. From the generalized Maxwell model, we reproduced the negative long-time tail, but with a slower $t^{-2}$ decay. This indicates the atoms become collective through the propagation of elastic modes in supercooled liquids. The slower decay predicted by this model is believed to be caused by the underestimation of the hydrodynamic effect.

The authors thank Ting Wang and Hideyuki Mizuno for valuable discussions. The authors are also grateful to the computer resources provided by Konstanz Scientific Computer Cluster and Jülich Super-computing Center. We acknowledge financial support from the German Academic Exchange Service (DAAD) for funding through the DLR-DAAD program under Grant No. 131.
[1] J. P. Hansen and I. R. Macdonald, Theory of Simple Liquids, 3rd ed. (Academic, New York, 2005).

[2] D. J. Evans and G. Morriss, Statistical Mechanics of Nonequilibrium Liquids, 2nd ed. (Cambridge University, Cambridge, England, 2008).

[3] B. J. Alder, D. M. Gass, and T. E. Wainwright, J. Chem. Phys. 53, 3813 (1970).

[4] B. J. Alder and T. E. Wainwright, Phys. Rev. Lett. 18, 988 (1967).

[5] R. F. Fox, J. Chem. Phys. 64, 5307 (1976).

[6] C. Morkel, C. Gronemeyer, W. Gläser, and J. Bosse, Phys. Rev. Lett. 58, 1873 (1987).

[7] B. J. Alder and T. E. Wainwright, Phys. Rev. A 1, 18 (1970).
[8] M. A. van der Hoef, D. Frenkel, and A. J. C. Ladd, Phys. Rev. Lett. 67, 3459 (1991).

[9] S. R. Williams, G. Bryant, I. K. Snook, and W. van Megen, Phys. Rev. Lett. 96, 087801 (2006).

[10] M. H. Ernst and A. Weyland, Phys. Lett. A 34, 39 (1971).

[11] F. Höfling and T. Franosch, Phys. Rev. Lett. 98, 140601 (2007).

[12] L. Berthier and G. Biroli, Rev. Mod. Phys. 83, 587 (2011).

[13] R. Yamamoto and A. Onuki, Phys. Rev. E 58, 3515 (1998).

[14] M. D. Ediger, Annu. Rev. Phys. Chem 51, 99 (2000).

[15] M. H. Ernst, J. Machta, J. R. Dorfman, and H. van Beijeren, J. Stat. Phys. 34, 477 (1984). 
[16] J. Machta, M. H. Ernst, H. van Beijeren, and J. R. Dorfman, J. Stat. Phys. 35, 413 (1984).

[17] C. Ganter and W. Schirmacher, Phys. Rev. B 82, 094205 (2010).

[18] A. Fiege, T. Aspelmeier, and A. Zippelius, Phys. Rev. Lett. 102, 098001 (2009).

[19] D. Chakraborty, Eur. Phys. J. B 83, 375 (2011).

[20] M. H. J. Hagen, I. Pagonabarraga, C. P. Lowe, and D. Frenkel, Phys. Rev. Lett. 78, 3785 (1997).

[21] R. D. Mountain, J. Chem. Phys. 102, 5408 (1995).

[22] H. Mizuno and R. Yamamoto, Phys. Rev. Lett. 110, 095901 (2013).

[23] J. Farago, H. Meyer, and A. N. Semenov, Phys. Rev. Lett. 107, 178301 (2011).

[24] W. Kob and H. C. Andersen, Phys. Rev. E 51, 4626 (1995).
[25] S. Plimpton, J. Comp. Phys. 117, 1 (1995).

[26] U. Balucani and Zoppi, Dynamics of the Liquid State (Oxford University, Oxford, 1994).

[27] U. Balucani, R. Vallauri, and T. Gaskell, Phys. Rev. A 35, 4263 (1987).

[28] D. Levesque and L. Verlet, Phys. Rev. A 7, 1690 (1973).

[29] T. Gaskell and S. Miller, J. Phys. C: Solid State Phys. 11, 3749 (1978).

[30] C. Donati, S. C. Glotzer, P. H. Poole, W. Kob, and S. J. Plimpton, Phys. Rev. E 60, 3107 (1999).

[31] M. H. Ernst and J. R. Dorfman, J. Stat. Phys. 12, 311 (1975).

[32] Y. Pomeau, Phys. Rev. A 7, 1134 (1973).

[33] R. E. Ryltsev and N. M. Chtchelkatchev, J. Chem. Phys. 141, 124509 (2014). 